\title{
An Enhanced Approach for Synchronization in WSN
}

\author{
Sanjay Madaan \\ M.Tech, Department of \\ Computer Science \& \\ Engineering, \\ GZS PTU Campus, Bathinda
}

\author{
Dinesh Kumar \\ Assistant Professor, \\ Department of Computer \\ Science \& Engineering, \\ GZS PTU Campus, Bhatinda
}

\author{
Robin Khurana \\ Assistant Professor, \\ Department of Computer \\ Science \& Engineering, \\ IGCE, Abhipur Mohali
}

\begin{abstract}
Small, low cost, low power and multifunctional sensor are developed as a result of improvement in microelectromechanical (MEMS) technology. Sensor's are connected via wireless medium and are used to observe various aspects of the physical world. Data sensed by these nodes needs to be ag-glomerated using data fusion, which requires clocks of nodes to be synchronized with each other. Existing approaches of clock synchronization are not designed keeping wireless sensor networks in mind. So there is a need to extend or redesign and develop a new way to synchronize the wireless sensor networks that is best suited to the specific needs of a sensor-network application. In this paper, we introduced a new approach of synchronization of nodes in WSN by enhancing the CSMA/CA protocol (slotted ALOHA). A Master Node, which is assumed to be synchronized with GPS (Global positioning System), deployed in the network. The nodes in a cluter synchronized their local clock with master node using NTP. The proposed solution features minimal energy consumption, minimal packet loss and achieve better throughput.
\end{abstract}

\section{Keywords}

Clock Synchronization, WSN, CSMA/CA, GPS.

\section{INTRODUCTION}

A wireless sensor network (WSN) is a distributed network consisting, in general, of a large number of sensor nodes, which are densely deployed over a wide geographical region to track a certain physical phenomenon. A wireless sensor network is a promising novel tool for observing natural phenomena at large scale or high resolution. Random deployment of wireless sensors node in terrains or during disasters relief operations is feasible, as the position of sensor node need not to be predetermined. [1]

Ad-hoc deployment of thousands of sensors, each capable of detecting conditions such as sound, light, temperature, presure and movement of certain objects and with a short-range wireless communication channel creates a sensor rich "smart environment" [2]. The unique feature of wireless sensor networks is the collaborative effort of sensor nodes to perform tasks such as data fusion, detection and measurement. Processing the raw data in to the meaningful data by performing simple computations and transmit the partially processed data is the major task performed by a sensor node.

Wireless sensor networks can be widely used in applications such as medical [3], environmental [4-6],scientific, industrial, military [7], and home networks [8]. Specifically, WSNs enable doctors/psychologists to identify predefined symptoms by monitoring the physiological data of patients remotely. In military, WSNs finds its application to detect nuclear,biological, and chemical attacks and presence of hazardous materials, prevent enemy attacks by means of alerts when enemy aircrafts are spotted.[9]

As the wireless sensor node power with limited energy and when deployed in the area where human perception is not possible, the recharge or replacement of energy resources might be impossible. Sensor node lifetime shows a strong dependence on battery lifetime. If the sensor nodes may get die due to limited battery then the chances of network breakage increases and results in the problem to collect data (temperature, humidity etc) of that particular area. As the dual role, data originator and data router, is performed by the sensor node the malfunctioning of a few nodes can cause significant topological changes and might require rerouting of packets and reorganization of the network. Hence, energy conservation takes on additional importance. Many techniques are proposed for energy saving, clustering (the process of dividing the nodes in to clusters) is one of them.

The cluster heads are elected periodically such that members of a cluster can communicate with their cluster heads. These cluster heads send data received from its members to a base station. The multi clustering can also be used. The cluster head should have to be rotated for the balancing of energy and then there will be equal load on every node. The energy consumption can be reduced.

A critical piece of infrastructure for any distributed system is time synchronization. The wireless sensor network make use of synchronized time in following listed tasks [1]:

- To integrate a time-series of proximity detections into a velocity estimate [10];

- To measure the time-of-flight of sound for localizing its source [11];

- $\quad$ To distribute a beam forming array [12];

- To suppress redundant messages [13];

As the major task of the WSN is to observe the natural phenomena at large scale, without accurate time information, sensed data often loses while transmitting. We can say that time is a first-class citizen in wireless sensor network. Some of the sensor networks estimate the location of an event, simply by using trilateration on an acoustic signal [14].

In addition, time synchronization is significant in WSN as sensor network protocols make use of time in various forms. In order to avoid interference in transmission, Media access control (MAC) using TDMA needs accurate time information. Similarly, to save energy, sensor network protocols often employ advanced duty-cycling schemes, and turn off their radio if not needed [3]. Time synchronization in the sensor 
network helps the nodes to access the channel in a reserved slot only. In addition, accurate time results in shortening the necessary wake-up guard times and helps to save energy.

The hardware clock exhibits a large skew even though they are well calibrated at deployment. So, these hardware clocks can usually not be used directly, as they suffer from severe drift. To allow for an accurate common time, nodes need to exchange messages from time to time, constantly adjusting their clock values.

In this paper, we argue that the need for energy efficiency, the different requirements across sensor network application are not found in conventional distributed systems. In this paper, we proposed a new scheme for synchronization of sensor clocks using NTP approach.

The work presented in this paper will focus on the performance enhancement of CSMA/CA protocol using NTP. The impact on the performance of WSN in terms of throughput, packet loss, energy consume will be analysed. The rest of the paper is organized as follows. Section 2 briefly explains the objective of the work. Section 3 presents a brief Literature Survey of the proposed protocols for Clustering and Synchronization of WSN. Section 4 represents the problem formulation. Section 5 shows the experimental results when the proposed approach is simulated using NS2 simulator.

\section{OBJECTIVE OF THE WORK}

The main objectives of this work are:

1. To enhance the CSMA/CA protocol to increase reliability of wireless sensor network

2. To reduce packet loss in the network by synchronizing the cluster head nodes with the use of NTP approach.

3. To implement the proposed novel technique and compare the results with the existing problem graphically.

\section{LITERATURE SURVEY}

From the last few decades, various techniques and protocols has been proposed to enhance the improvement of WSN. One of the approaches is to divide the network in to clusters. Clustering is an effective approach for organizing the network into a connected hierarchy, load balancing, and prolonging network lifetime. Based on the concept of forming the clusters at once or reform the cluster after every scheduled time, clustering protocols are classified into static and dynamic. In static clustering, clusters are formed once, forever and role of the cluster head is scheduled among the nodes in a cluster where as in dynamic clustering the time is divided into rounds and clustering is performed in the beginning of each round.

Another approach is to synchronize the logical clocks of the sensor nodes in the network. If the clocks are synchronized, packet collision is less.

Here different approaches for clock synchronization and clustering are discussed.

In [15], Philipp Sommer et. al. addressed an issue of locality in multi-hop clock synchronization. Global clock skew minimization, means to minimize the skew between any two far-away nodes, is the main objective in clock synchronization.

Multi-hop clock synchronization protocols such as FTSP [16] are designed to optimize the global skew. As FTSP and similar protocols work on a spanning tree, synchronizing nodes in the tree with their parents, and ultimately with the root of the tree. In spanning tree, as the error propogate down differently on the different paths of the tree, the neighbouring nodes which are not closely related in the tree will not be synchronized well.

A new protocol, Gradient Time Synchronization Protocol (GTSP), for synchronization is proposed that excels primarily at local clock synchronization. GTPS rely on local clock information and is completely distributed requiring no reference node or tree constructions. The results of proposed algorithm, performed on a testbed of Mica2 sensor nodes, and by simulations, shows that the proposed approach results in a better average synchronization between neighbours while still maintaining a tolerable global skew.

M.D Jovanovic et.al in [17] introduces a TFMAC, a hybrid MAC protocol in which each sensor node is equipped with a single half-duplex transceiver with multiple-frequency support. TFMAC allows the different sensor nodes to transmit on different channels simultaneously by combining multiple channels into a traditional TDMA scheme. The author represents the simulated results, which reflects that the proposed multiple-channel protocol provides higher maximum throughput and smaller average packet delay in respect to the basic single-channel TDMA scheme.

In [18], Fan Xiangning et. al presents energy-LEACH and multi-hop protocols making the process of selection the cluster head in next round energy based. Communication mode from single hop to multi-hop between cluster head and sink is improved using multi-hop protocol. In [18] hundreds and thousands of wireless sensors are dispersed that collects and transmit data and cluster heads are elected (turn-wise)out of the sensors to transmit the data collected to base station. Simulation result shows that selecting a cluster head in earlier mentioned way make the protocol energy efficient.

In [19], B.A Bakr et. al, proposed an optimal energy-saving spare management, including spare selection and named it LEACH-SM protocol (modified form of prominent LEACH protocol). LEACH-SM adds the spare selection phase to LEACH. In this author presented a quantitative comparison of energy consumption and WSN lifetime for both mentioned protocols.

A new architecture called HARP (Hierarchical Adaptive and Reliable Routing Protocol) proposed by F.J. Atero, et. al builds inter cluster and intra-cluster hierarchical trees, which are further optimized to save power. This architecture is scalable and finds it application both in homogeneous and heterogeneous wireless sensor networks. Efficient link fault tolerance and node mobility management are the features provided by addition of a recovery slot in the scheduling scheme. Furthermore, to optimize and balance the energy consumption in the network, a new cluster heads election formulation and its associated data gathering protocol (sHARP) is proposed [20].

A comparative study of clustering techniques and cluster quality of a single criterion cluster heads election and cluster formation in WSN is discussed in [21]. In order to show how the cluster formation helps to prolong the network lifetime: the HEED, LEACH and Energy-based LEACH protocols are simulated and their performance are compared in terms of the number of cluster head generated, cluster size, cluster head distribution, scalability and coverage. Further, scalability aspects in the presence of advanced nodes in the network and its effect on the network lifetime are studied in [21]. 
A new approach for cluster heads election that improved network lifetime in the presence of advanced nodes, proposed in [21], shows that lifetime of the network can be improved as compared to having more homogeneous nodes in the network by just introducing fraction of advanced nodes in the network.

Hybrid Clustering Approach (HCA) presented by Peyman Neamatollahi, et al. in [22] is based on the concept of performing the clustering in the next round, whenever a cluster head consumes a pre specified part of its energy. That means, clustering is performed on demand. A well known distributed clustering protocol, HEED, is used as baseline example to evaluate the efficiency of proposal. Simulation results show that significant energy is saved using HCA.

In order to achieve low energy consumption in large wireless sensor networks, deployed nodes have to organize themselves to avoid unnecessary sensor and transceiver operations. In [23], the proposed Hex-MASCLE algorithms combine advantages of temporal and spatial network fragmentation. As compare to previous approaches, the shapes of the basic cells are given by regular hexagons, similar to honeycombs. In the present work, a significant improvement in network lifetime is achieved by hexagon-based clustering with self-healing abilities. The proposed algorithms are more fault-tolerant against localization errors, overhead and achieve fairly uniform cluster head distribution across the network.

An enhanced version of hierarchical routing protocol LEACH, named E-LEACH is proposed in [24]. In the E-LEACH algorithm, cluster head selection is random and the round time for the selection is fixed. In this, remnant power of the sensor nodes and changes the round time depends on the optimal cluster size. Increase in $40 \%$ network lifetime is achieved by proposed protocol as compared to LEACH algorithm, is the result of simulation. No doubt, LEACH protocol prolongs the network lifetime in contrast to plane multi-hop routing and static routing, it still has problems. The proposed protocol eliminates the drawback, cluster heads communicate with the base station in single-hop mode, of LEACH protocol in largescale wireless sensor networks. In LEACH, the nodes with low remnant energy have the same priority to be a cluster head as the node with high remnant energy.

In [25], the author investigated the performance of four different slotted multi-access protocols for the single-packet medium access problem. The analytical comparison of transient Markov chain analysis for p-persistent slotted Aloha, slotted Aloha with constant and binary exponential back-off window, and slotted CSMA with constant back-off through the use of flow equations that captured the network dynamics as a function of time is presented. Expected delay and energy consumption per node are the parameters on the basis of which performance of above mentioned protocol is measured and suggested the most appropriate protocol that is dependent on these parameters.

In [26], a new approach is proposed which results in increment of network throughput by $10 \%$ and approximately doubles the network lifetime as compared to slotted ALOHA wireless sensor network without advertisements. The proposed scheme, cross-layer Frame Rate Adaption Scheme reduces transmission overhead by not adding protocol information to be transmitted with data bits, when compared to other medium access methods.

\section{PROBLEM FORMULATION}

Synchronization protocols proposed by researchers from past few decades are well suited for wired networks and challenges posed by wireless sensor network are different and manifold. The most important differences are summarized here.

- As sensor nodes are cheaper and smaller in size, the nodes can be widely deployed.

- Self-configuration and robustness.

- As nodes are widely deployed and it is impossible to provide a power source to each sensor in such a vast network, energy conservation is a very important concern.

- Further, the small sizes of sensors restrict the amount of energy that can be stored and procured.

Taking the above four points in to concern and the previous discussion in section 2, energy conserve and time synchronization is the two important issues in WSN.

Let us consider the following scenario, in which the sensor nodes are grouped together to form a cluster. Clustering of nodes in used to reduce the battery utilization of nodes as the cluster-head takes part in communication mostly. The senor nodes in one cluster send the sensed data to cluster-head using single-hop communication.

As in figure 1, the cluster-heads of cluster 1 and 3 send the sensed data to cluster-head of cluster 2. As the clocks of cluster-heads are not synchronized, this results in packet loss at cluster-head of cluster 2 .

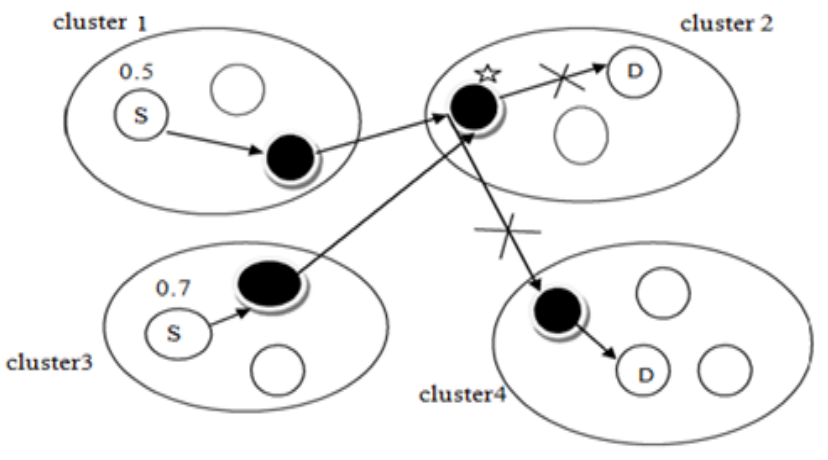

Figure 1: A Network with non-synchronous nodes and packet collision

In the proposed solution, a special node is deployed in the network (master node), which is synchronized with GPS (Global Positioning System). This master node sends the information to all the cluster heads and cluster heads distribute this information to all cluster members. The clocks are synchronized using the NTP approach.

Let us consider another scenario, the reflects the proposed solution. The clocks are synchronized with master node, which uses the Offset Delay Estimation Method [10].

NTP involves a hierarchical tree of time servers. The primary server at the root synchronizes with the UTC (Universal Coordinated Time). The next level contains secondary servers, which act as a backup to the primary server. At the lowest level is the synchronization subnet which has the clients [11]. 
As shown in figure 2, the shaded nodes in each cluster acts as a cluster-head, which synchronize its clock with master node and the nodes in a cluster synchronizes their clock with respective cluster-heads.

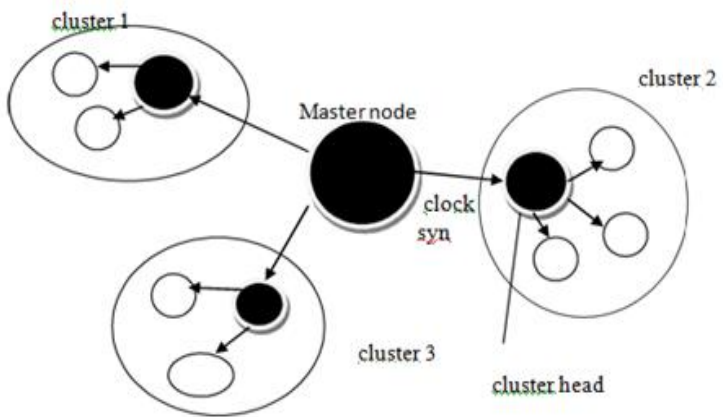

Figure 2: Node synchronous process

Now in the sensor network all the nodes are synchronized and slotted aloha is applied to the network. According to the slotted aloha the node which wants to transmit the data reserves the time slots. All the nodes get the different slots and they transmit their data according to their turn. Hence, result in less packet collision and reduction in packet loss.

\section{EXPERIMENTAL RESULTS}

To evaluate the performance of the proposed algorithm we implemented the scenario using ns2 simulator and compare the parameters (energy, packet loss, and throughput) of the network based on synchronized and non-synchronized network.

The nodes are deployed in an area of $800 * 800 \mathrm{~m}^{2}$. The whole network is divided in to four hexagonal shaped clusters, similar to honeycombs. Each of the clusters consists of four nodes and one cluster-head. A master node is deployed i.e for synchronization and sink node for connecting the network with outside world. The cluster-head performs dual role as data originator and data router. As LEACH protocol prolongs the network lifetime in contrast to plane multi-hop routing and static routing. In $\mathrm{LEACH}$, the nodes with low remnant energy have the same priority to be a cluster head as the node with high remnant energy.

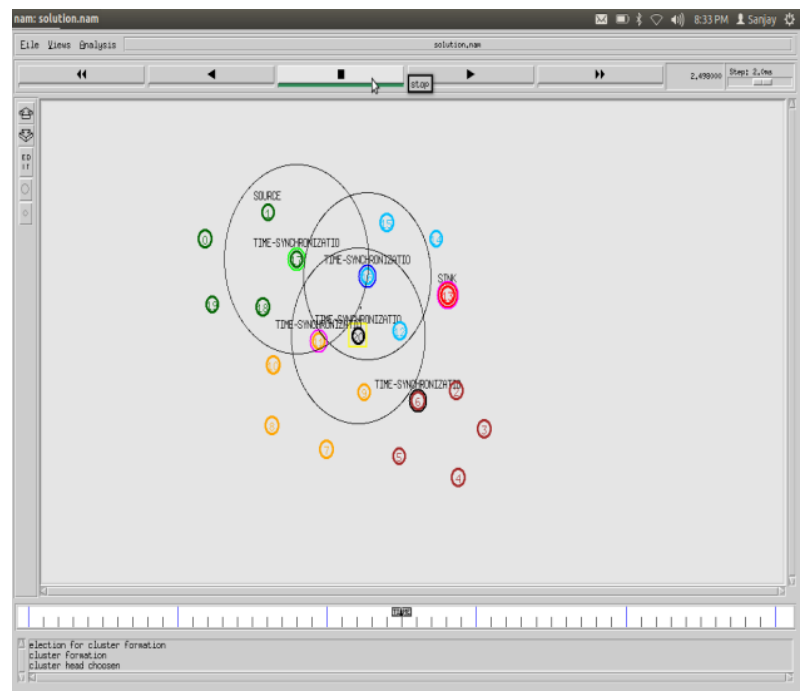

Figure 3: Synchronization
The slotted ALOHA is used for channel sensing and efficiency of the slotted ALOHA increases when cluster heads clocks are timely synchronized. If there is no collision between the packets, then there is no need to transmit the packets again. As the most of the energy is consumed in the communication and if the communication reduced to again transmit the packets then the battery consumption of the nodes can also be reduced. Hence there is a less energy consumption.

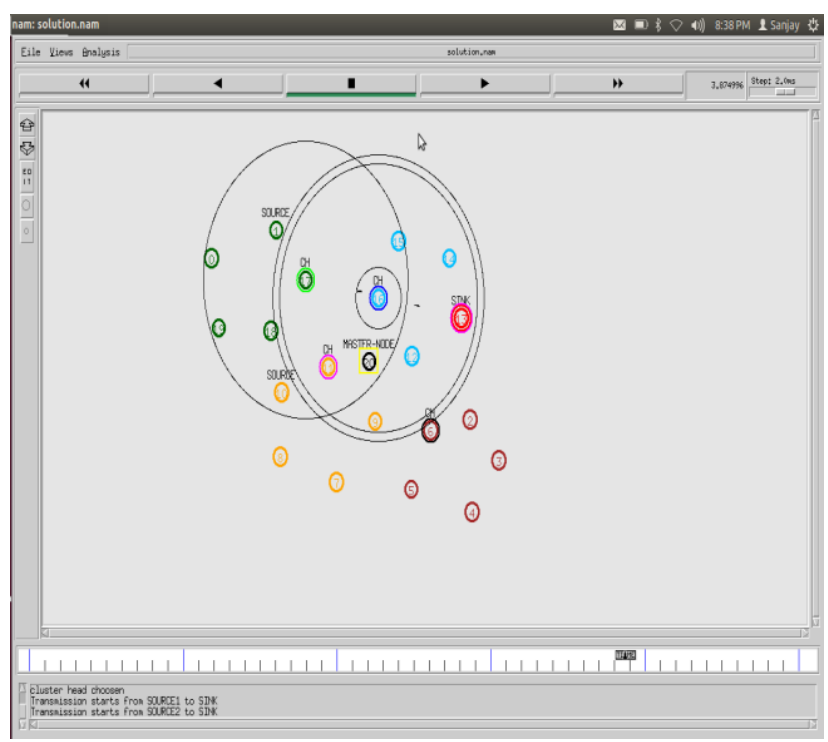

Figure 4: Synchronized Communications

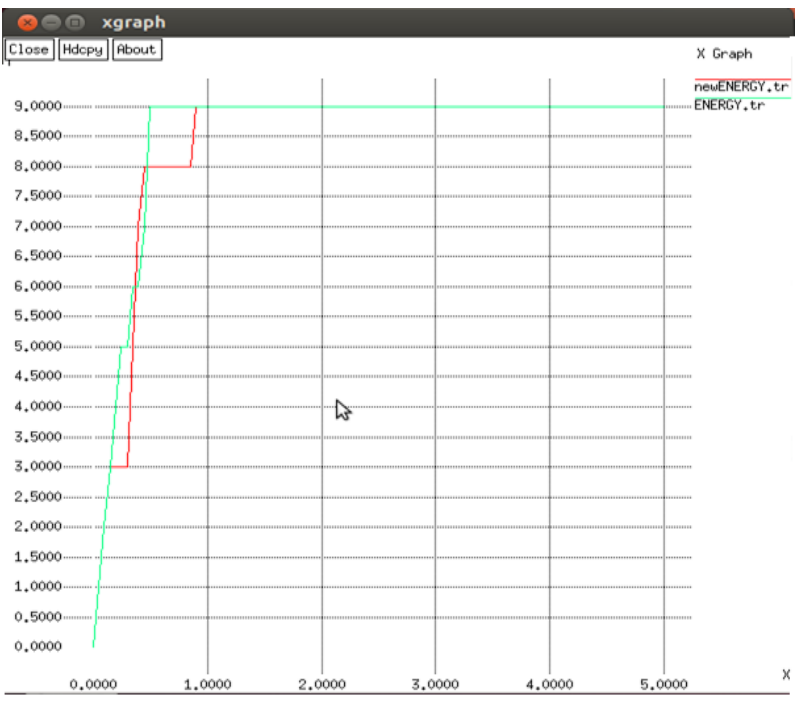

Figure 5: Energy Graph

Above figure shows the graph of energy consumption in the network w.r.t time. The graph reflects that energy consumption in proposed technique (red line) is low that that of the previous technique (green line). This is because of the fact that when the clock of nodes is synchronized, packet loss is less and so the packet retransmission. 


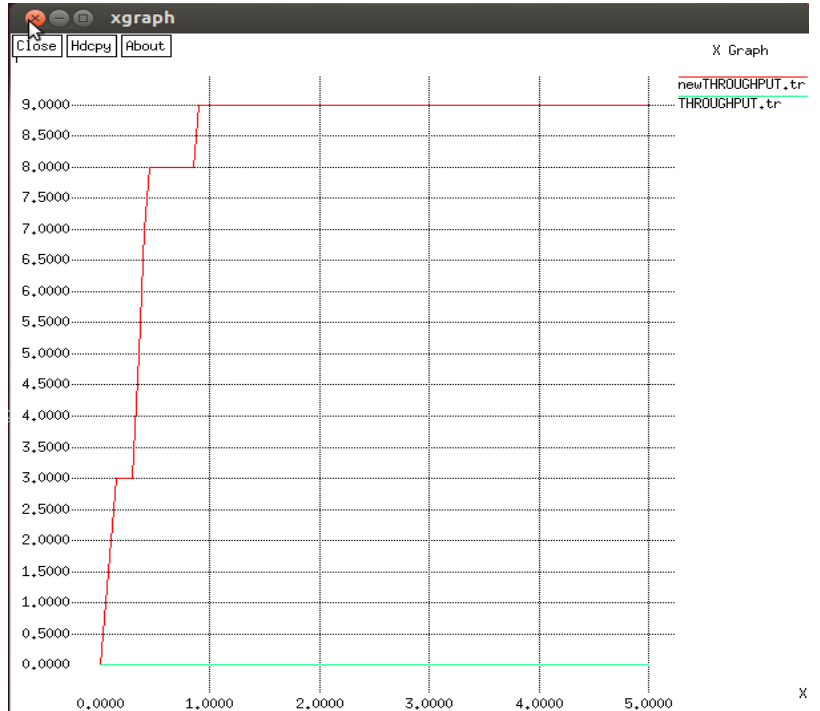

Figure 6: Throughput Graph

Above figure shows the comparison of throughput of the network in proposed technique and previous technique. The efficiency of the slotted ALOHA is enhanced with the clock synchronization. The throughput of the network is enhanced through the use of new proposed technique because the packet loss in the network is reduced.

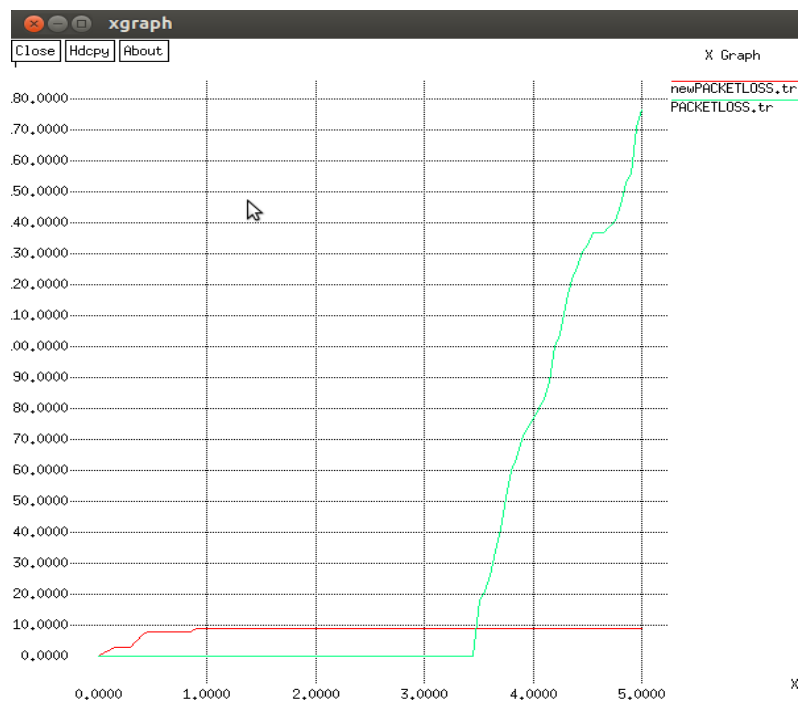

Figure 7: Packet Loss Graph

As in the previous technique, as the clocks of the cluster heads are not timely synchronized, the packet loss is high. The concept of synchronizing the clock of cluster heads with the master node, packet loss. In above figure, green line and red line shows the packet loss in previous and proposed technique respectively.

\section{CONCLUSION}

Our main focus in this paper is to synchronize the clocks of the sensors nodes in a network and to compare the results with unsynchronized network. The simulated results show the network efficiency in terms of reduced packet loss, better throughput and less energy consumption. The number of nodes taken in this scenario is limited to 5 per cluster, including cluster-head. In future, the proposed approach of synchronization can be simulated for unlimited number of nodes. Further, the packet delay can be studied which arises when packet travels over a wireless channel.

The latency of the channel can be decomposed in to four distinct components: Send Time, Access Time, Propagation Time, and Receive Time.

\section{REFERENCES}

[1] Jeremy Elson and Deborah Estrin, "Time Synchronization for Wireless Sensor Networks", International Parallel and Distributed Processing Symposium (IPDPS) pp. 41-46, Workshop on Parallel and Distributed Computing Issues in Wireless Networks and Mobile Computing, April 2001, San Francisco, CA, USA.

[2] Akyildiz, I; Su, W.; Sankarasubramanian, Y; Cayirci, E. Wireless sensor networks: a survey. Comput. Netw. 2002, 4, 393-422.

[3] Johnson, P. et al. Remote continuous physiological monitoring in the home. J. Telemed. Telecare 1996, 2, 107-113.

[4] CENS: Monitoring of marine microorganisms Homepage.

http://www.cens.ucla.edu/Research/Applications/momm. htm (accessed November 10, 2008).

[5] CENS: Seismic monitoring and structural response Homepage. http://www.cens.ucla.edu/Research/ Applications/seismicmonitor.htm (accessed on November 15, 2008)

[6] Szewczyk, R.; Osterweil, E.; Polastre, J.; Hamilton, M.; Mainwaring, A.; Estrin, D. Habitat monitoring with sensor networks. Commun. ACM 2004, 6, 34-40.

[7] Distributed Surveillance Sensor Network. ONR SPAWAR Systems Center, San Diego Homepage. http:// www.spawar.navy.mil/robots/undersea/dssn/dssn.html (accessed November 10, 2008)

[8] Johnson, P. et al. Remote continuous physiological monitoring in the home. J. Telemed. Telecare 1996, 2, 107-113.

[9] Ill-Keun Rhee, Jaehan Lee, Jangsub Kim, Erchin Serpedin,* and Yik-Chung Wu "Clock Synchronization in Wireless Sensor Networks: An Overview" Sensors 2009, 9, pg. no. 56-85.

[10] A. Cerpa, J. Elson, D. Estrin, L. Girod, M. Hamilton, and J. Zhao. Habitat monitoring: Application driver for wireless communications technology. In Proceedings of the 2001 ACM SIGCOMM Workshop on Data Communications in Latin America and the Caribbean, April 2001.

[11] L. Girod. Development and characterization of an acoustic rangefinder. Technical Report 00-728, University of Southern California, Department of Computer Science, March 2000.

[12] K. Yao, R. Hudson, C. Reed, D. Chen, and F. Lorenzelli. Blind beamforming on a randomly distributed sensor array system. IEEE Journal of Selected Areas in Communications, 16(8):1555-1567, Oct 1998. 
[13] C. Intanagonwiwat, R. Govindan, and D. Estrin. Directed diffusion: A scalable and robust communication paradigm for sensor networks. In Proceedings of the Sixth Annual International Conference on Mobile Computing and Networking, pages 56-67, Boston, MA, Aug. 2000. ACM Press.

[14] G. Simon, M. Maróti, Á. Lédeczi, G. Balogh, B. Kusy, A. Nádas, G. Pap, J. Sallai, and K. Frampton. Sensor network-based countersniper system. In SenSys '04: Proceedings of the 2nd international conference on Embedded networked sensor systems, 2004.

[15] Philipp Sommer, Roger Wattenhofer "Gradient Clock Synchronization in Wireless Sensor Networks" IPSN'09, April 13-16, 2009, San Francisco, California, USA.

[16] M. Maróti, B. Kusy, G. Simon, and Á. Lédeczi.The Flooding Time Synchronization Protocol. In SenSys '04: Proceedings of the 2nd international conference on Embedded networked sensor systems, 2004.

[17] Milica D. Jovanovic1 and Goran Lj. Djordjevic1 "TFMAC: Multi-channel MAC Protocol for Wireless Sensor Networks" 2011 IEEE.

[18] Fan Xiangning and Song Yulin "Improvement on LEACH Protocol of Wireless Sensor Network" 2007 IEEE
[19] Bilal Abu Bakr and Leszek Lilie "A Quantitative Comparison of Energy Consumption and WSN Lifetime for LEACH and LEACH-SM" 2011 IEEE.

[20] F.J. Atero, J.J. "A Low Energy and Adaptive Architecture for Efficient Routing and Robust Mobility Management in Wireless Sensor Networks" 2011 IEEE.

[21] Dahlila P. Dahnil, et al."Energy-Efficient Cluster Formation in Heterogeneous Wireless Sensor Networks: A Comparative Study" Feb.2011.

[22] Peyman Neamatollahi, et al."A Hybrid Clustering Approach for Prolonging Lifetime in Wireless Sensor Networks" 2011 IEEE.

[23] Jakob Salzmann, et al. "Hex-MASCLE - Hexagon based Clustering with Self Healing Abilities" 2011 IEEE.

[24] Jia Xu, et.al "Improvement of LEACH protocol for WSN" 2012 IEEE.

[25] Kiran Yedavalli and Bhaskar Krishnamachari, "Analysis of Slotted Multi-Access Techniques for Wireless Sensor Networks".

[26] Lucas D. P. Mendes et.al "Lifetime Analysis of a Slotted ALOHA-based Wireless Sensor Network using a Crosslayer Frame Rate Adaptation Scheme”, IEEE, 2011. 